\title{
Recent CLEO Results on Tau Hadronic Decays
}

\author{
J.E. Duboscq ${ }^{\mathrm{a}}$ for the CLEO collaboration \\ ${ }^{a}$ Wilson Laboratory, Cornell University, Ithaca NY 14850, USA \\ I outline recent results using CLEO3 data involving the decay of the $\tau$ to three charged hadrons and a neutrino, \\ as well as an investigation of the structure of the decay $\tau \rightarrow K K \pi \nu$ and the Wess-Zumino term in this decay.
}

\section{The Decay of the $\tau$ to Three Charged Hadrons and a neutrino}

When the $\tau$ lepton decays to 3 charged hadrons and a neutrino, the predominant decay mode involves three charged pions. Occasionally this decay will include one, two or three kaons in the final state. Each of these modes is useful for understanding different physics topics. The decay $\tau^{-} \rightarrow K^{-} \pi^{+} \pi^{-} \nu$ is important in extracting the strange spectral function as well the strange quark mass and the CKM matrix element $V_{u s}$. The decay $\tau^{-} \rightarrow K^{-} K^{+} \pi^{-} \nu$ allows one to probe the Wess Zumino term in the effective lagrangian. Finally, the decay $\tau \rightarrow K^{-} K^{+} K^{-} \nu$ is as yet unobserved.

In this analysis we use a subset of almost 3 million $\tau$ pairs produced at the CESR $e^{+} e^{-}$collider at or near the $\Upsilon(4 S)$ resonance, analyzed with the CLEO3 3 detector. Since the predominant decay of the $\tau$ to 3 charged hadrons usually involves pions, we use the CLEO3 RICH detector to identify Kaons with high efficiency while rejecting pion fakes. This is combined with the detector's $d E / d x$ capabilities to identify pions and kaons with efficiencies in the $\approx 90 \%$ region and reject fakes at the $\approx 3 \%$ level up to momenta of almost $2 \mathrm{GeV} / \mathrm{c}$. These efficiencies and fake rates are extracted from data using the decay chain

\footnotetext{
*I am thankful for the fine efforts of the CESR staff for creating the many collisions which make this work possible. I would also like to thank the National Science Foundation for its essential support. The conference organizers merit many thanks for their very enjoyable conference. Presented at the 8th International Workshop on Tau-Lepton Physics, Nara, Japan, Sept 2004. To appear in Nuclear Physics B - Proceedings Supplements.
}

Table 1 Candidate event yields, estimated $\tau$ and $q \bar{q}$ backgrounds, and efficiencies for $\tau \rightarrow 3 h \nu$

\begin{tabular}{|c|c|c|c|c|}
\hline Mode & Data & $\tau$ bgd & $q \bar{q}$ bgd & $\epsilon(\%)$ \\
\hline$\pi \pi \pi$ & 43543 & $3207 \pm 57$ & $152 \pm 12$ & $10.27 \pm 0.08$ \\
\hline$K \pi \pi$ & 3454 & $1475 \pm 38$ & $57 \pm 8$ & $11.63 \pm 0.12$ \\
\hline$K K \pi$ & 932 & $86 \pm 9$ & $19 \pm 4$ & $12.48 \pm 0.11$ \\
\hline$K K K$ & 12 & $4 \pm 2$ & $0.4 \pm 0.6$ & $9.43 \pm 0.10$ \\
\hline
\end{tabular}

$$
D^{*} \rightarrow D^{0} \pi, D^{0} \rightarrow K \pi .^{2}
$$

At CLEO near the $\Upsilon(4 S)$ resonance, $\tau$ 's are produced back to back with substantial momentum. This allows us to select $\tau$ events by looking for events with 1 track recoiling against 3 tracks, with the event hemispheres identified using the event thrust axis. The single track hemisphere is required to be consistent with either a $\tau$ decay to an electron, muon, single pion, or $\rho$ with an unseen neutrino. Events with extra showers are rejected to cut down on the feed-through from $\tau \rightarrow 3 h \pi^{0} \nu$ events. Missing momentum and visible energy cuts reduce 2 photon fusion background events. Also, events consistent with the production of a $K_{S}^{0}$ are rejected in the $K \pi \pi$ mode. Events are simulated using KORALB, JETSET and GEANT, while particle identification efficiencies are taken from data as noted above.

The breakdown of the analysis results are shown in Table 1 The largest $\tau$ backgrounds are feedthroughs from $\tau \rightarrow 3 h\left(\pi^{0}\right) \nu$ modes. The

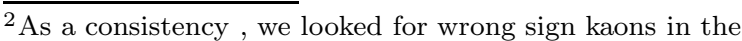
decay $\tau^{-} \rightarrow K^{+} \pi^{-} \pi^{+} \nu$, and found that data and Monte Carlo estimations agreed.
} 


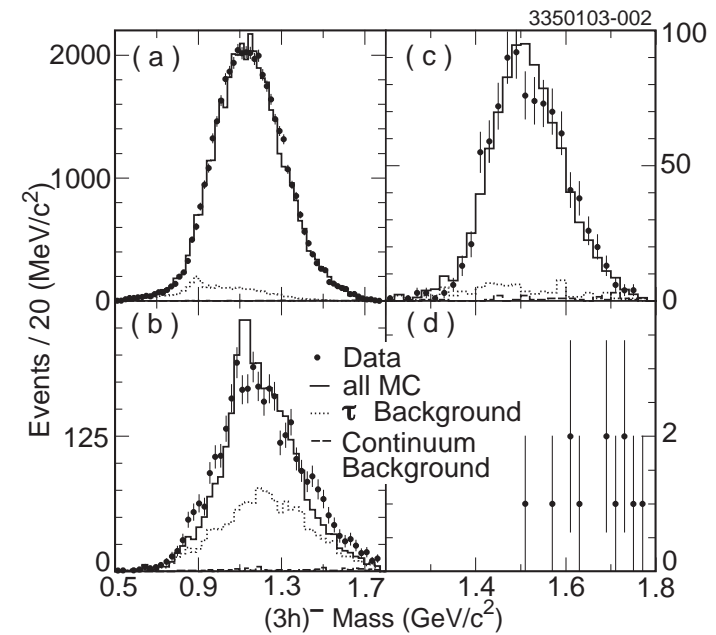

Figure 1. Three hadron masses from data (points). Tau pair Monte Carlo yields are shown as short dashes, $q \bar{q}$ Monte Carlo as long dashes; the sum of the background and signal MC is the solid histogram. (a) $\pi \pi \pi$ mass, (b) $K \pi \pi$ mass, (c) $K K \pi$ mass, (d) $K K K$ mass

feed-across is determined from the Monte Carlo, except in the $K K K$ signal mode in which the data is used. The resulting three body mass plots are shown in Fig 1 For each of these channels the two body mass structure is shown in Fig 2 These plots show very good agreement between data and Monte Carlo. The tunings used for the $\pi \pi \pi$ and $K \pi \pi$ components are those reported at TAU02 [5]. The channel $K K \pi$ had its substructure tuned to the data. This required decreasing the $K^{*}$ contribution, increasing the $\rho^{\prime}$, and removing the $\rho^{\prime \prime}$ component entirely.

The dominant systematic error in this study comes from particle identification efficiency $(3 \%)$ and particle identification fakes (ranging from $0.1 \%$ to $12 \%$.) These were determined using the both the $D^{*}$ sample referred to above, as well as a search for the wrong sign decay $\tau^{-} \rightarrow K^{+} \pi^{-} \pi^{-} \nu$. A systematic error of $2 \%$ was estimated for the tuning of the $K K \pi$ substructure.

Final branching ratio results are found to be:

$$
\begin{aligned}
B\left(\tau^{-} \rightarrow \pi^{-} \pi^{+} \pi^{-} \nu\right) & =9.13 \pm 0.05 \pm 0.46 \% \\
B\left(\tau^{-} \rightarrow K^{-} \pi^{+} \pi^{-} \nu\right) & =0.384 \pm 0.014 \pm 0.038 \%
\end{aligned}
$$

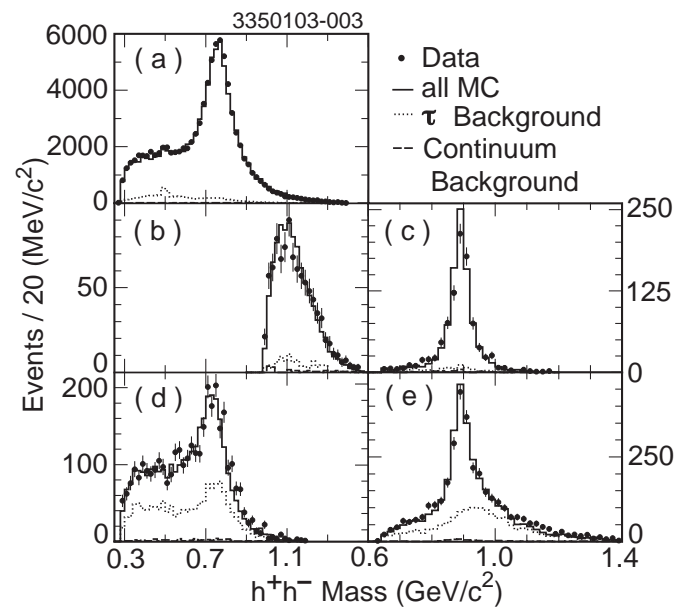

Figure 2. Two body substructure of the 3 body mass samples for oppositely charged hadrons. Data are shown as points, Tau pair Monte Carlo yields are shown as short dashes, $q \bar{q}$ Monte Carlo as long dashes; the sum of the background and signal MC is the solid histogram. (a) $\pi \pi$ mass in $\pi \pi \pi$ ( 2 entries per event), (b) $K K i$ mass in $K K \pi$, (c) $K \pi$ mass in $K K \pi$, (d) $\pi \pi$ mass in $K \pi \pi$, (e) $K \pi$ mass in $K \pi \pi$ 


$$
\begin{aligned}
B\left(\tau^{-} \rightarrow K^{-} K^{+} \pi^{-} \nu\right) & =0.155 \pm 0.006 \pm 0.009 \% \\
B\left(\tau^{-} \rightarrow K^{-} K^{+} K^{-} \nu\right) & <3.7 \times 10^{-5} @ 90 \% C L
\end{aligned}
$$

The $\pi \pi \pi$ result is the first in which all three pions are explicitly identified. The $K \pi \pi$ result is consistent with the previous OPAL result of $0.360 \pm 0.082 \pm 0.048 \%$ [6] and the CLEO2 [4] result of $0.346 \pm 0.023 \pm 0.056 \%$. The result is higher than the ALEPH reported value [7] of $0.214 \pm 0.037 \pm 0.029 \%$. The $K K \pi$ result is the most precise currently available, while the $K K K$ limit is the most stringent. This work has been published in 8

\section{The Structure of $\tau \rightarrow K K \pi \nu$ and the Wess Zumino Anomaly}

In the simplest picture of $\tau$ decays, vector (axial) currents produce an even (odd) number of pseudoscalars in the final state. The Wess $\mathrm{Zu}-$ mino anomaly term in the effective Lagrangian allows for a parity flip and can cause a violation of this simple rule. The golden mode for this mechanism $\tau \rightarrow \eta \pi \pi^{0} \nu$ contains no axial term and has been previously observed by CLEO ( 9$]$ ). The decay $\tau \rightarrow K K \pi \nu$ is expected to have both axial and vector contributions. Extracting the vector component allows one to examine the WZ term in this decay.

The $\tau$ decay to 3 hadrons and a neutrino can be expressed as a product of a leptonic current and a hadronic current [10. The hadronic current can be expanded into a sum over 4 form factors $\left(F_{i}\right)$ : $J=\Sigma f_{i}\left(q_{1}, q_{2}, q_{3}\right) F_{i}\left(s_{1}, s_{2}, Q\right)$. In this expression, the $q_{i}$ are the individual hadron four momenta, the $s_{i}$ are the pairwise hadron momenta, and $Q$ is the total four momentum of the hadronic system. The $f_{i}$ are kinematic terms. The $F_{1}$ and $F_{2}$ are the axial vector terms. The $F_{3}$ term is the $\mathrm{W}-\mathrm{Z}$ vector term and its corresponding kinematic term $f_{3}$ is $i \epsilon^{\alpha \beta \gamma} q_{1 \alpha} q_{2 \beta} q_{3 \gamma}$. This $\epsilon$ invokes the parity flip that allows the WZ mechanism to operate. (The $F_{4}$ term corresponds to the negligible scalar current.)

In order to extract these terms, we integrate over the unobserved neutrino direction. The two remaining Euler angles are kinematically deter- mined at CLEO. With this it turns out that the differential decay rate can be expressed as: $d \Gamma(\tau \rightarrow K K \pi) / d Q^{2} d s_{1} d s_{2} \propto W_{A}\left(F_{1}, F_{2}\right)+$ $W_{B}\left(F_{3}\right)$. The $W_{B}$ term expresses the strength of the $\mathrm{W}-\mathrm{Z}$ anomaly. Note that there is no interference between the $W_{A}$ and $W_{B}$ terms, and the extraction of these terms is possible by using only subcomponent masses and the $Q^{2}$ evolution of the decay.

In order to actually do this fit, we need to assume some model for the physics involved. We have used the following modification of the model in 11112: For the $F_{1}$ term, we allow the decay to proceed through $a_{1} \rightarrow \rho^{\left({ }^{\prime}\right)} \pi, \rho^{\left({ }^{\prime}\right)} \rightarrow$ $K K$. The $F_{1}$ term thus is proportional to $B W_{a 1}\left(Q^{2}\right) \times\left(B W_{\rho}\left(s_{2}\right)+\beta_{\rho} B W_{\rho}^{\prime}\left(s_{2}\right)\right)$, where BW denotes a Breit Wigner function. For the $F_{2}$ term, the decay proceeds through $a_{1} \rightarrow$ $K^{*} K, K^{*} \rightarrow K \pi$. The $F_{2}$ term is proportional to $R_{F} B W_{a_{1}}\left(Q^{2}\right) B W_{K^{*}}\left(s_{1}\right)$. For the $\mathrm{W}-\mathrm{Z}$ term, we parameterize the decay as occurring either through the channels $\rho^{\left({ }^{\prime},{ }^{\prime \prime}\right)} \rightarrow K^{*} K, K^{*} \rightarrow$ $K \pi$, or through $\rho^{\left({ }^{\prime},{ }^{\prime \prime}\right)} \rightarrow \omega \pi, \omega \rightarrow K K$. This leads to $F_{3} \propto R_{B}^{1 / 2}\left(B W_{\rho}\left(Q^{2}\right)+\lambda B W_{\rho^{\prime}}\left(Q^{2}\right)+\right.$ $\left.\delta B W_{\rho^{\prime \prime}}\left(Q^{2}\right)\right) \times\left(B W_{\omega}\left(s_{2}\right)+\alpha B W_{K^{*}}\left(s_{1}\right)\right)$

In the above there are 5 real parameters to fit: $R_{F}, R_{B}, \lambda, \delta$, and $\alpha$. Note that these fits are only used to model the decay and do not necessarily correspond to the correct physics. These five parameters are extracted from fits to the $K K \pi, K \pi$ and $K K$ mass plots.

The data used for this analysis corresponds to a superset of that of the previous analysis, comprising some 7 million $\tau$ pairs at CLEO3. The cuts used are the same as in the $\tau \rightarrow 3 h \nu$ analysis above, and result in 2255 signal events with an estimated $256 \pm 16 \pm 46$ background events. The absolute branching ratio obtained with this sample is consistent with that given above.

To extract the values of the five parameters we perform an unbinned extended maximum likelihood fit including a background term. The probability distribution function that is fit is a product of the individual PDFs for $K K \pi, K K, K \pi$. We use the best known values of the parameters for each Breit Wigner term.

Fig 3 shows the results of the fit for $K K \pi, K \pi$ 
and $K K$. Also shown on the plots are the WZ (vector) component and the axial component. It is clear that the $\mathrm{W}-\mathrm{Z}$ term is prominent in the fit - the result is that the partial width from $\mathrm{W}-\mathrm{Z}$ is: $\Gamma_{W Z} / \Gamma_{T o t}=55 \pm 8.4 \pm 4.9 \%$. The best fit parameters are given in Table 2

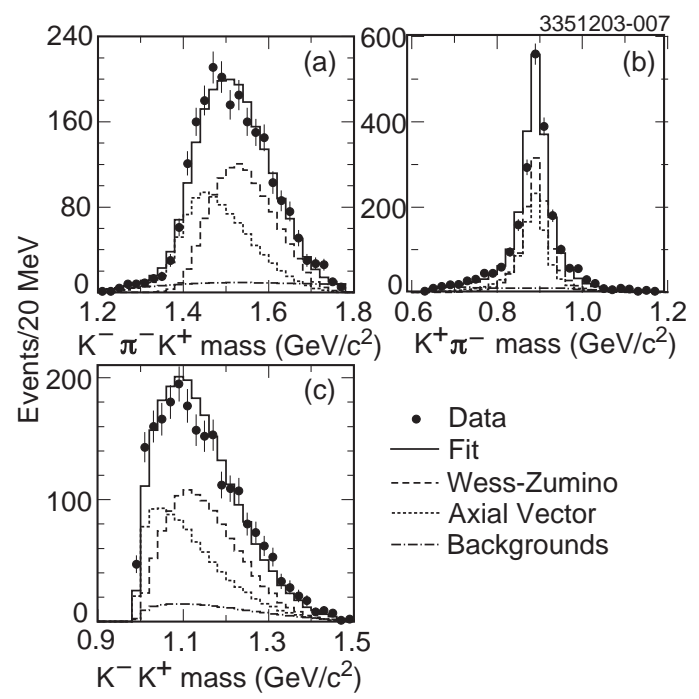

Figure 3. Fit projections (solid lines), data (points), and contributions from the W-Z term (dashed) and the axial term (dotted), as well as all backgrounds (dot-dashed) for the $K K \pi \mathrm{W}-\mathrm{Z}$ analysis. (a) $K K \pi$ mass projection, (b) $K^{+} \pi^{-}$ mass projection, (c) $K^{-} K^{+}$mass projection

The substructure of this fit can be expressed in the context of our modified Kuhn \& Mirkes model. The decay is found to be dominated by the $K^{*} K$ mode, with approximately equal $\mathrm{W}-\mathrm{Z}$ and axial components. Explicitly,

$$
\begin{aligned}
R_{W Z}^{\omega \pi} & =3.4 \pm 0.9 \pm 1.0 \% \\
R_{\text {Axial }}^{\rho^{(\prime)} \pi} & =2.50 \pm 0.8 \pm 0.4 \% \\
R_{W Z}^{K^{*} K} & =60.8 \pm 8.5 \pm 6.0 \% \\
R_{\text {Axial }}^{K^{*} K} & =46.8 \pm 8.4 \pm 5.2 \%
\end{aligned}
$$

These fractions do not add up to $100 \%$ because of interference terms in the intermediate states.
Table 2

W-Z Fit results

\begin{tabular}{|c|c|}
\hline$\alpha$ & $0.471 \pm 0.060 \pm 0.034$ \\
\hline$\lambda$ & $0.314 \pm 0.073 \pm 0.080$ \\
\hline$\delta$ & $0.101 \pm 0.020 \pm 0.156$ \\
\hline$R_{B}$ & $3.23 \pm 0.26 \pm 1.90$ \\
\hline$R_{F}$ & $0.98 \pm 0.15 \pm 0.36$ \\
\hline \hline$\Gamma_{W Z} / \Gamma_{T o t}$ & $55 \pm 8.4 \pm 4.9 \%$ \\
\hline
\end{tabular}

We extract the value of the branching fraction $B\left(a_{1} \rightarrow K^{*} K\right)=2.2 \pm 0.5 \%$ and this is found to be consistent with expectations from the CLEO analysis for the decay $\tau \rightarrow 3 \pi^{0} \nu$. Note that the axial component determined directly here is much smaller than that which has been found by Aleph's CVC estimate $\left(94_{-8}^{+6} \%\right)$ [13] using data from the DM1 and DM2 collaborations. Note that our fit does have a floating value of $R_{B}$ which might contribute to the observed difference. This work has been published in 14.

\section{Summary}

In this talk, we have presented the first direct measurement of $B\left(\tau-\rightarrow \pi^{-} \pi^{+} \pi^{-} \nu_{\tau}\right)$. We have also presented a measurement of $B(\tau-\rightarrow$ $\left.K^{-} \pi^{+} \pi^{-} \nu_{\tau}\right)$ consistent with previous work from OPAL and CLEO, but higher than the result found by ALEPH. We've set the most stringent limit on the branching fraction of the decay of a $\tau$ to 3 kaons and a neutrino, and have presented the best precision on $B\left(\tau \rightarrow K^{-} K^{+} \pi^{-} \nu_{\tau}\right)$. We have also presented the first study of the WZ anomaly and the axial component of the decay $\tau^{-} \rightarrow K^{-} K^{+} \pi^{-} \nu_{\tau}$.

\section{REFERENCES}

1. R. Ammar et al (CLEO Collaboration), Phys Rev D 49,5701 (1994)

2. Y.Kubota et al (CLEO Collaboration), Nucl. Instrum. Methods Phys. Res., Sect. A 320, 66 (1992) ; T. Hill, Nucl. Instrum. Methods Phys. Res. Sect. A 418, 32 (1998).

3. G. Viehhauser, CLEO III Operation,Nucl. Instrum. Methods A 462, 146 (2001). 
4. S.J. Richichi et al (CLEO Collaboration), Phys. Rev. D 60, 112002 (1999)

5. A. Weinstein, hep-ex/0210058

6. G.Abbiendi et al (OPAL Collaboration) Euro. Phys. Journal C 13, 197 (2000.)

7. R. Barate et al (ALEPH Collaboration), Euro.Phys. Journal C1, 65 (1998)

8. R. Briere et al (CLEO Collaboration), Phys. Rev. Lett. 90, 181802 (2003).

9. M. Artuso et al (CLEO Collaboration), Phys.Rev.Lett 69, 3278 (1992)

10. Kuhn, Mirkes, Z.PhysC56, 661(1992).

11. Decker et al, Z.Phys.C58, 445(1996).

12. Finkemeir \& Mirkes, Z,Phys.C69, 24(1996).

13. R. Barate et al (ALEPH Collaboration), Euro. Phys. Journal C 11 (1999) 599-618

14. T.E. Coan et al (CLEO Collaboration), Phys. Rev. Lett. 92, 232001 (2004) 